\title{
Presence in virtual social networks and health promoting behaviors of medical students
}

\author{
Mahnaz Pouresmaeil $^{1} \cdot$ Akram Shahrokhi $^{2} \cdot$ Kazem Hosseinzadeh $^{3} \cdot$ Arash Ziapour $^{4}$ (D) $\cdot$ Fatemeh Rahimi $^{5}$
}

Received: 20 July 2019 / Accepted: 23 January 2020

(C) Springer-Verlag GmbH Germany, part of Springer Nature 2020

\begin{abstract}
Aims Students are significantly present in virtual social networks and spend a considerable amount of time in this space. An increase in the number of users of virtual social networks has led to the emergence of a new lifestyle, known as the virtual lifestyle, which can affect the daily activities of students, especially their health behaviors. Therefore, this study is aimed to determine the relationship of virtual social networks with health-promoting behaviors among the medical students of Qazvin University of Medical Sciences.

Method In this descriptive-analytic study, 318 medical students were included using the stratified random sampling technique. By means of a 52-item health-promoting behavior scale (Walker et al.) and a researcher-made questionnaire, the use of virtual social networks was studied. The data were analyzed in SPSS (V.22) software by Kruskal-Wallis, Mann-Whitney, Pearson's and Spearman correlation coefficients, independent t-test and one-way ANOVA at a significance level of 0.05 .

Results The use of virtual social networks was high (66\%), and the mean score of health-promoting behaviors of students was moderate $(135.32 \pm 19.35)$. The data analysis showed a significant relationship between the aspects of responsibility for health, nutrition status and interpersonal relationships and the use of virtual social networks $(P<0.05)$. A meaningful correlation was also found between the use of virtual social networks and demographic characteristics of students including marital status, age, employment status and residence $(P<0.05)$. However, no significant relationship was obtained with other demographic variables, and no statistically meaningful association was observed between health-promoting lifestyle and any of the demographic variables.

Conclusion According to the findings of the study, it seems that the presence of students in virtual social networks can be an opportunity and, through creating special student groups under the supervision of professors working in the field of health promotion, it is possible to publish scientific content in these networks to take a step toward promoting the health of students.
\end{abstract}

Keywords Virtual social network $\cdot$ Health-promoting behavior $\cdot$ Medical student

\section{Introduction}

Social networks, as a type of social media, are a new generation of internet websites, in which users can virtually exchange information on a common topic and create online groups. Social networks consist of individuals, groups and their relationships (Eslami 2012).

Virtual social networks provide the possibility of creating online personal accounts in a specified space, in which people can introduce themselves to and communicate with others and

\author{
Akram Shahrokhi \\ a.shahrokhi@qums.ac.ir \\ Mahnaz Pouresmaeil \\ mahnzpou@gmail.com \\ Kazem Hosseinzadeh \\ kazemhosseinzadeh@gmail.com \\ Arash Ziapour \\ arashziapoor@gmail.com \\ Fatemeh Rahimi \\ f-rahimi@razi.tums.ac.ir
}

Health Education and Promotion, Qazvin University of Medical Sciences, Qazvin, Iran

2 Faculty, Nursing \& Midwifery School, Qazvin University of Medical Sciences, Qazvin, Iran

3 Health Education and Promotion, Nursing \& Midwifery School, Qazvin University of Medical Sciences, Qazvin, Iran

4 Health Education and Health Promotion, Health Institute, Kermanshah University of Medical Sciences, Kermanshah, Iran

5 Department of Health Education and Promotion, School of Public Health, Tehran University of Medical Sciences, Tehran, Iran 